\title{
Behavioral assessment in patients with disorders of consciousness: gold standard or fool's gold? ${ }^{\text {is }}$
}

\author{
Joseph T. Giacino ${ }^{1,2, *}$, Caroline Schnakers ${ }^{3}$, Diana Rodriguez-Moreno ${ }^{4}$, \\ Kathy Kalmar ${ }^{1}$, Nicholas Schiff ${ }^{5}$ and Joy Hirsch ${ }^{4}$ \\ ${ }^{1} J F K$ Johnson Rehabilitation Institute, Edison, NJ, USA \\ ${ }^{2}$ New Jersey Neuroscience Institute, Edison, NJ, USA \\ ${ }^{3}$ Coma Science Group, Cyclotron Research Centre, University of Liege, Liège, Belgium \\ ${ }^{4}$ fMRI Research Center, Columbia University, New York, USA \\ ${ }^{5}$ Weill Medical College of Cornell University, New York, USA
}

\begin{abstract}
In the absence of "hard" neurophysiologic markers, the burden of proof for establishing conscious awareness in individuals who sustain severe brain injury lies in behavioral assessment. Because behavior represents indirect evidence of consciousness, reliance on behavioral markers presents significant challenges and may lead to misdiagnosis. Detection of conscious awareness is confounded by numerous factors including fluctuations in arousal level, difficulty differentiating reflexive or involuntary movement from intentional behavior, underlying sensory and motor impairments, and medication side effects. When an ambiguous behavior is observed, the onus falls to the clinician to determine where along the continuum of unconsciousness to consciousness, it lies. This paper (1) summarizes the current diagnostic criteria for coma, the vegetative state, and the minimally conscious state, (2) describes current behavioral assessment methods, (3) discusses the limitations of behavioral assessment techniques, (4) reviews recent applications of functional neuroimaging in the assessment of patients with disorders of consciousness, and (5) concludes with a case study that illustrates the disparity between behavioral and functional neuroimaging findings that may be encountered in this population.
\end{abstract}

Keywords: disorders of consciousness; vegetative state; minimally conscious state; assessment scales; brain injury; rehabilitation

Progress in intensive care has increased the number of patients who survive severe acute brain injury. Most recover from coma within the first 2 weeks after the insult, others require more

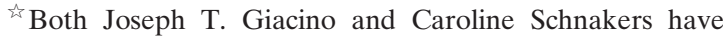
contributed equally to this study.

*Corresponding author.

Tel.: 732-205-1461; Fax: 732-632-1584;

E-mail: jgiacino@solarishs.org
}

time and pass through different stages before fully or partially recovering consciousness. One of the most challenging problems facing clinicians is understanding the natural history of recovery from severe brain injury. In clinical practice, it is often difficult to detect unambiguous signs of consciousness in patients with limited behavioral repertoires. This complication is reflected in frequent misdiagnoses (Andrews et al., 1996; Childs et al., 1993). Assessment of residual brain 
function in severely brain-injured patients is difficult because motor responses may be impaired, inconsistent, or easily exhausted. For these reasons, diagnostic criteria and standardized behavioral scales have been developed to facilitate the assessment of consciousness in patients recovering from coma. Other techniques such as functional neuroimaging can provide additional information to gauge cognitive processing and aid in diagnostic assessment. This paper (1) summarizes the current diagnostic criteria for coma, the vegetative state (VS), and the minimally conscious state (MCS), (2) describes current behavioral assessment methods, (3) discusses the limitations of behavioral assessment techniques, (4) reviews recent applications of functional neuroimaging in the assessment of patients with disorders of consciousness (DOC), and (5) concludes with a case study that illustrates the disparity between behavioral and functional neuroimaging findings that may be encountered in this population.

\section{Diagnostic criteria}

It is essential to distinguish DOC's such as coma, VS, and MCS (see Table 1) as there are important differences in recovery course and outcome. These disorders must also be distinguished from the locked-in syndrome (LIS) and brain death as these conditions have overlapping features, although neither represents a DOC.

\section{Coma}

Plum and Posner (1966) defined coma as a pathological state marked by severe and prolonged dysfunction of vigilance and consciousness. This state results from global brain dysfunction (most often due to diffuse axonal injury following traumatic brain injury), or from a lesion limited to brainstem structures involving the reticular activating system. The distinguishing feature of coma is the continuous absence of eye-opening (spontaneously or following stimulation). There is no evidence of visual fixation or pursuit, even after manual eye-opening. No voluntary motor behavior is observed and behavioral responses are limited to reflex activity only. Brain electrical activity is observed, albeit characterized by slow frequency bands (i.e., mostly delta and theta activity). This state must last at least $1 \mathrm{~h}$ to be differentiated from a transient DOC such as syncope, acute confusion, or delirium. Prolonged coma is rare as this condition usually resolves within 2-4 weeks, most often evolving into VS or MCS.

\section{Vegetative state}

The term "vegetative state" denotes reactivation of autonomic functions (e.g., cardio-vascular, respiratory, and thermoregulation functions) with concomitant reemergence of the sleep-wake cycle (i.e., periods of spontaneous eyes opening). VS often results from trauma-induced bi-hemispheric injury involving the white matter or from bilateral lesions in the thalamus with sparing of the brainstem, hypothalamus, and basal ganglia (Plum and Posner, 1983). Behaviorally, there is no response to verbal order and, although moaning may occur, there is no intelligible speech (The Multi-Society Task Force on PVS, 1994). Infrequently, behaviors such as inappropriate smiling, crying, or grimacing, and even randomly produced single words have been reported in patients diagnosed with VS (Schiff et al., 1999; Working Party of the Royal College of Physicians, 2003). With serial multimodal assessment, the probability that these behaviors are not voluntary or goal directed can be further investigated, although not proven. When this state lasts 1 month or more, the term "persistent VS" has been applied (The Multi-Society Task Force on PVS, 1994). In view of the high rate of recovery of consciousness after 1 month (Choi et al., 1994; Giacino and Kalmar, 1997), and well-documented cases of late recovery (Childs and Mercer, 1996), the American Congress of Rehabilitation Medicine has recommended that the term "persistent VS" be abandoned in favor of documenting the cause of the VS (trauma, anoxia) and the length of time post-onset, as both carry prognostic information (American Congress of Rehabilitation Medicine, 1995). When VS lasts more than 3 months after non-traumatic brain injury, and 1 year following 
Table 1. Diagnostic criteria for brain death, coma, vegetative and minimally conscious states, and locked-in syndrome

\begin{tabular}{|c|c|c|}
\hline Consciousness level & Diagnostic criteria & Reference(s) \\
\hline \multirow[t]{4}{*}{ Brain death } & No arousal/eye-opening & \multirow{4}{*}{$\begin{array}{l}\text { Medical Consultants on the } \\
\text { Diagnosis of Death (1981) }\end{array}$} \\
\hline & No behavioral signs of awareness & \\
\hline & Apnea & \\
\hline & Loss of brain functions (brainstem reflexes) & \\
\hline \multirow[t]{6}{*}{ Coma } & No arousal/eye-opening & \multirow[t]{6}{*}{ Plum and Posner (1966) } \\
\hline & No behavioral signs of awareness & \\
\hline & Impaired spontaneous breathing & \\
\hline & Impaired brainstem reflexes & \\
\hline & No vocalizations & \\
\hline & $>1 \mathrm{~h}$ & \\
\hline \multirow[t]{14}{*}{ Vegetative state } & Arousal/spontaneous or stimulus-induced eye opening & \multirow{7}{*}{$\begin{array}{l}\text { The Multi-Society Task Force } \\
\text { on PVS (1994) }\end{array}$} \\
\hline & No behavioral signs of awareness & \\
\hline & Preserved spontaneous breathing & \\
\hline & Preserved brainstem reflexes & \\
\hline & No purposeful behaviors & \\
\hline & No language production or comprehension & \\
\hline & $>1$ month: persistent vegetative & \\
\hline & Compatible & \multirow{7}{*}{$\begin{array}{l}\text { Working Party of the Royal } \\
\text { College of Physicians (2003) }\end{array}$} \\
\hline & Grimaces to pain & \\
\hline & Localization to sounds & \\
\hline & Atypical but compatible & \\
\hline & Visual fixation & \\
\hline & Response to threat & \\
\hline & Inappropriate single words & \\
\hline \multirow[t]{11}{*}{ Minimally conscious state } & Arousal/spontaneous eye-opening & \multirow[t]{11}{*}{ Giacino et al. (2002) } \\
\hline & Fluctuating but reproducible behavioral signs of awareness & \\
\hline & Response to command & \\
\hline & Environmentally contingent emotional/motor responses & \\
\hline & Object localization and manipulation & \\
\hline & Sustained visual fixation and pursuit & \\
\hline & Intelligible verbalization & \\
\hline & Intentional but unreliable communication & \\
\hline & Emergence from MCS & \\
\hline & Functional communication & \\
\hline & Functional object use & \\
\hline \multirow[t]{5}{*}{ Locked-in syndrome } & Arousal/spontaneous eye-opening & \multirow{5}{*}{$\begin{array}{l}\text { American Congress of } \\
\text { Rehabilitation Medicine (1995) }\end{array}$} \\
\hline & Preserved cognitive functions & \\
\hline & Communication via eye gaze & \\
\hline & Anarthria & \\
\hline & Tetraplegia & \\
\hline
\end{tabular}

traumatic etiologies, VS can be considered "permanent" (The Multi-Society Task Force on PVS, 1994).

\section{Minimally conscious state}

MCS is characterized by the presence of inconsistent but clearly discernible behavioral signs of consciousness (Giacino et al., 2002).
Command-following, recognizable yes-no responses, and intelligible verbalizations represent the clearest evidence of conscious awareness. In contrast to patients in VS who may display random episodes of crying or smiling, in MCS, these behaviors occur in contingent relation to appropriate environmental triggers. Reemergence of visual pursuit appears to be an early behavioral marker of the transition from VS to MCS (Giacino 
and Whyte, 2005). Although behavior may fluctuate across examinations, at least one of these signs must be replicated within a given examination to meet the diagnostic criteria for MCS.

Regarding prognosis, the probability of functional recovery at 1 year following traumatic brain injury is significantly more favorable relative to VS (50\% vs. $3 \%$ attaining moderate disability). Some patients in MCS progress slowly while others remain in this condition permanently (Fins et al., 2007). It is also important to recognize that, unlike VS, clearly defined temporal parameters for recovery do not yet exist (Lammi et al., 2005), and there is wide heterogeneity in the degree of functional recovery ultimately attained. Emergence from MCS occurs when the patient is able to reliably communicate through verbal or gestural yes-no responses, or is able to demonstrate use of two or more objects (e.g., hairbrush, cup) in a functional manner (Giacino et al., 2002).

\section{Differential diagnosis}

Two additional conditions characterized by behavioral unresponsiveness must be differentiated from VS and MCS. In the first, consciousness is retained, while in the second, it is permanently lost.

\section{Locked-in syndrome}

LIS is marked by tetraplegia and anarthria in the setting of near-normal to normal cognitive function (American Congress of Rehabilitation Medicine, 1995). This state is caused by a lesion involving the ventral pons and, in $60 \%$ of cases, is due to basilar thrombosis. Because patients with LIS have spontaneous eyes opening, but are unable to speak or move the extremities, this state can be confused with VS because of the confluence of behavioral signs. On average, the diagnosis of LIS is not established until 2.5 months post-onset. There is evidence that family members tend to detect signs of consciousness (55\% of cases) prior to medical staff (23\% of cases) (Laureys et al., 2005a). Classic LIS consists of complete paralysis of the orobuccal musculature and all four extremities, however, vertical eyes movements are spared, allowing nonverbal communication through directional gaze. Perceptual functions are also usually spared as ascending corticospinal axons remain intact (American Congress of Rehabilitation Medicine, 1995). Bauer et al. (1979) have described multiple varieties of LIS, including the incomplete form in which there is residual motor activity (frequently, finger or head movement), and total LIS, in which there is complete immobility including both horizontal and vertical eye movements. Data on life expectancy suggest that some patients with LIS live 12 or more years post-onset. Surprisingly, chronic LIS patients rate their quality of life similarly to the healthy population (Laureys et al., 2005a). In the absence of other structural or functional brain abnormalities, patients with LIS are generally able to make independent decisions and communicate their preferences (Schnakers et al., 2008b; Smart et al., 2008).

\section{Brain death}

Brain death is a condition in which there is "irreversible unconsciousness with complete loss of brain function." It is marked by the presence of apnea and the lack of any behavioral response to the environment (Medical Consultants on the Diagnosis of Death, 1981). Generally, an electroencephalogram is completed to demonstrate an iso-electrical signal reflecting the absence of electrical brain activity. Transcranial Doppler studies reveal the absence of cerebral blood flow. Finally, functional imaging, using cerebral perfusion tracers and single photon emission tomography (SPECT), illustrate the "empty skull" sign in which the "whole brain" is inactive (Facco et al., 1998). After excluding brain dysfunction due to drug toxicity or hyperthermia, a final diagnosis can be established after 6-24 h.

\section{Behavioral assessment methods}

Twenty-five years ago, Plum and Posner (1983) noted that, "the limits of consciousness are hard to define satisfactorily and we can only infer the 
self-awareness of others by their appearance and their acts". As noted, behavioral observation remains the "gold standard" for detecting signs of consciousness in severely brain-injured patients. Preservation of arousal is a necessary but insufficient condition for consciousness (see Fig. 1). The search for consciousness rests on the demonstration of behavioral qualities that are distinct from simple reflexes. Behavioral assessment may not, however, definitively distinguish between behaviors associated with the state of arousal and those linked to conscious awareness. This dilemma is illustrated in the difficulty clinicians often have in differentiating reflexive eye blinks from eyeclosure to command. Additionally, consciousness may not be a static phenomenon and may be better conceptualized as a continuum. It is possible, for example, for a patient in coma to rapidly evolve into VS, gradually transition to MCS, and subsequently lapse back into VS (Giacino and Trott, 2004; Majerus et al., 2005).

\section{Behavioral scales}

Numerous behavioral rating scales have been developed and validated to assess level of

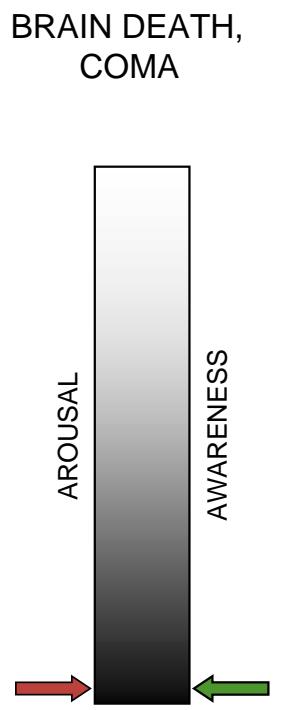

consciousness and establish diagnosis (Majerus et al., 2005). In this section, we briefly review instruments commonly used in the acute and rehabilitation settings.

\section{Acute setting}

The Glasgow Coma Scale (GCS) remains the most widely used scale in trauma and acute care settings. The GCS was the first validated rating scale developed to monitor level of consciousness in the intensive care unit (Teasdale and Jennett, 1974). This scale is relatively brief and can easily be incorporated into routine clinical care. It includes three subscales that address arousal level, motor function, and verbal abilities. Subscales scores are added and yield a total score ranging from 3 to 15. Despite its widespread use, the GCS has been criticized for variable inter-rater agreement and problems deriving scores in patients with ocular trauma, tracheostomy, or ventilatory support (McNett, 2007).

The Full Outline of UnResponsiveness (FOUR) scale was recently developed to replace the GCS to assess severely brain-injured patients in intensive care (Wijdicks, 2006; Wijdicks et al., 2005).

\section{LOCKED-IN SYNDROME}

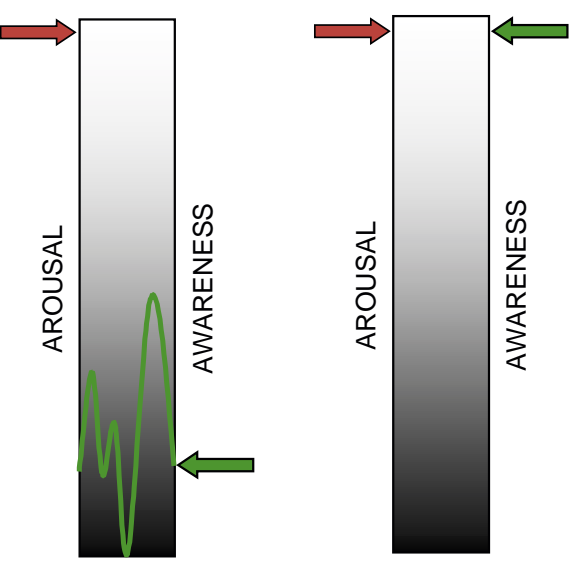

Fig. 1. Behavioral observation assesses two dimensions of consciousness: arousal and awareness. In brain death and coma, both dimensions are absent. In the vegetative state, arousal level is relatively preserved in the absence of signs of awareness. In the minimally conscious state, both dimensions are present although behavioral signs of awareness often fluctuate. In the locked-in syndrome, both dimensions are fully preserved despite complete loss of speech and motor functions. 
The scale is comprised of four subscales assessing motor and ocular responses, brainstem reflexes, and breathing. The total score ranges from 0 to 16 . Unlike the GCS, the FOUR does not assess verbal functions to accommodate the high number of intubated patients in intensive care. A score of 0 on the FOUR assumes the absence of brainstem reflexes and breathing and, therefore, helps to diagnose brain death. The scale also monitors recovery of autonomic functions and tracks emergence from VS. The FOUR is specifically designed to detect patients with LIS as it uses oculomotor commands that exploit vertical eye movements and eye blinks, both of which are preserved in LIS.

The Wessex Head Injury Matrix (WHIM) (Shiel et al., 2000) was developed to capture changes in patients in VS through emergence from posttraumatic amnesia. This tool is particularly sensitive to detecting changes in patients in MCS not captured by traditional scales such as the GCS (Majerus and Van der Linden, 2000). Shiel and collaborators longitudinally followed 97 severely brain-injured patients recovering from coma to create the WHIM. WHIM items were ordered according to the sequence of recovery observed in these patients. The 62-item WHIM's six sections assess arousal level and concentration, visual consciousness (i.e., visual pursuit), communication, cognition (i.e., memory and spatiotemporal orientation), and social behaviors. The WHIM score represents the rank of the most complex behavior observed.

The Sensory Modality Assessment and Rehabilitation Technique (SMART) (Gill-Thwaites, 1997) was developed to identify signs of consciousness observed during "sensory stimulations programs" intended to support cerebral plasticity and improve level of consciousness (Wood, 1991). The SMART assesses eight modalities including visual, auditory, tactile, olfactory and gustatory sensation, motor functions, communication, and arousal level. The SMART is a hierarchical scale consisting of five response levels ("absence of response" - Level 1; "reflex response" - Level 2; "withdrawal response" - Level 3; "localization response" Level 4; "discriminative response" - Level 5). The SMART has previously been shown to have very good validity and reliability in a population of 60 patients diagnosed as being in a VS or in a MCS (Gill-Thwaites and Munday, 2004).

The JFK Coma Recovery Scale (CRS) was originally developed by investigators from the JFK Johnson Rehabilitation Institute in 1991 (Giacino et al., 1991). The scale was revised and republished in 2004 as the JFK Coma Recovery Scale-Revised (CRS-R) (Giacino et al., 2004). The purpose of the CRS-R is to assist with differential diagnosis, prognostic assessment, and treatment planning in patients with DOC. The scale consists of 23 items that comprise 6 subscales addressing auditory, visual, motor, oromotor, communication, and arousal functions (see Table 2). CRS-R subscales are comprised of hierarchically arranged items associated with brain stem, subcortical, and cortical processes. The lowest item on each subscale represents reflexive activity while the highest items represent cognitively mediated behaviors. Scoring is standardized and based on the presence or absence of operationally defined behavioral responses to specific sensory stimuli. Psychometric studies indicate that the CRS-R meets minimal standards for measurement and evaluation tools designed for use in interdisciplinary medical rehabilitation. Adequate inter-rater and test-retest reliability have been established indicating that the CRS-R can be administered reliably by trained examiners and produces reasonably stable scores over repeated assessments. Validity analyses support use of the scale as an index of neurobehavioral function and have shown that the CRS-R is capable of discriminating patients in MCS from those in VS which is of critical importance in establishing prognosis and formulating treatment interventions (Schnakers et al., 2006, 2008a; Vanhaudenhuyse et al., 2008). Spanish, Portuguese, Italian, German, French, Dutch, Norwegian, and Danish translations of the CRS-R are available.

\section{Limitations of behavioral assessment}

Differentiating between MCS and VS can be challenging as voluntary and reflexive behaviors can be difficult to distinguish and subtle signs of consciousness may be missed (Majerus et al., 
Table 2. Coma Recovery Scale-Revised record sheet

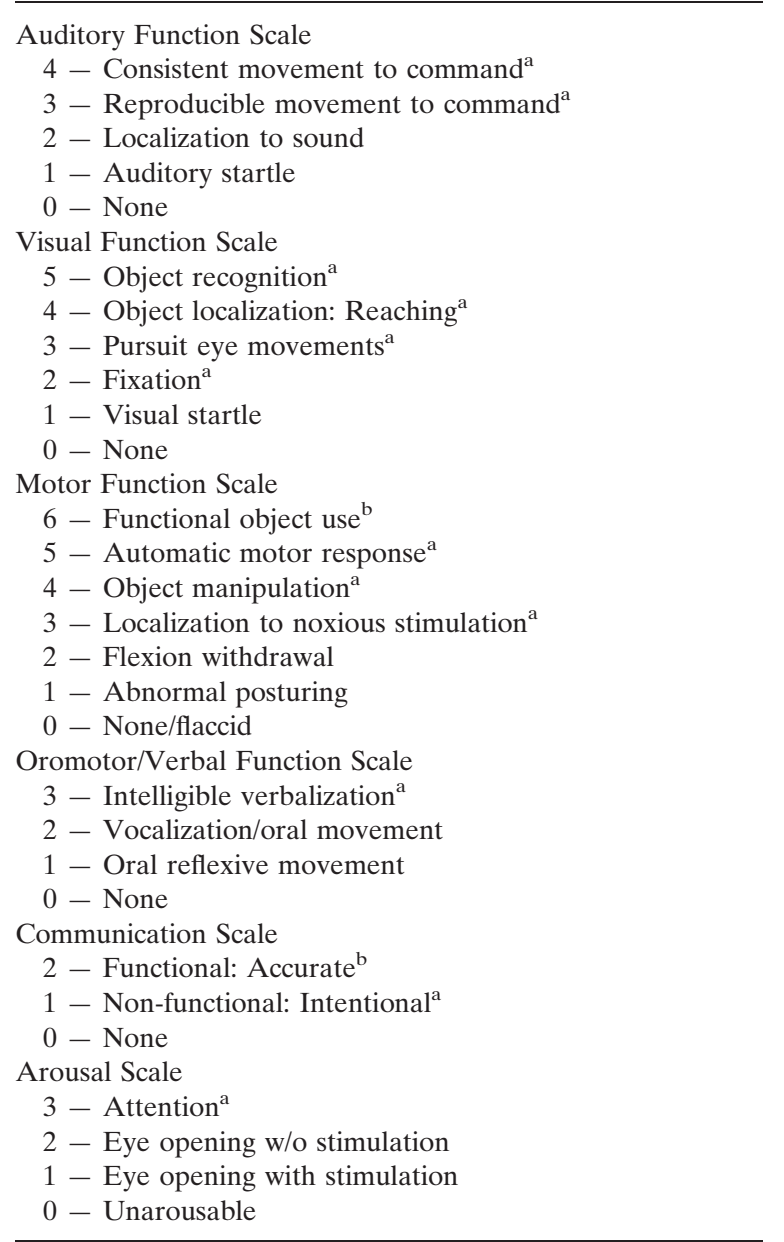

${ }^{\mathrm{a}}$ Denotes MCS.

${ }^{\mathrm{b}}$ Denotes emergence from MCS

2005). Prior studies have shown that $37-43 \%$ of patients with DOC are erroneously diagnosed with VS (Andrews et al., 1996; Childs et al., 1993). The recent development of diagnostic criteria for MCS (Giacino et al., 2002) would reasonably be expected to reduce the incidence of misdiagnosis relative to the rates reported before these criteria were established (Jennett, 2005). However, a recent study found that $41 \%$ of patients believed to be in VS were misdiagnosed. This study also found that the majority of cases with an uncertain diagnosis were in MCS (89\%), not in VS. Another $10 \%$ diagnosed with MCS had actually emerged from this condition (Schnakers et al., 2009).
The high rate of misdiagnosis reported by Schnakers and collaborators likely reflects different sources of variance. Variance in diagnostic accuracy may result from biases contributed by the examiner, patient, and environment. Examiner error may arise when the range of behaviors sampled is too narrow, response-time windows are over or under-inclusive, criteria for judging purposeful responses are poorly defined or not adhered to, and examinations are conducted too infrequently to capture the full range of behavioral fluctuation. The use of standardized rating scales offers some protection from these errors, although failure to adhere to specific administration and scoring guidelines may jeopardize diagnostic accuracy (Schnakers et al., 2009). The second source of variance concerns the patient. Fluctuations in arousal level, fatigue, subclinical seizure activity, occult illness, pain, cortical sensory deficits (e.g., cortical blindness/deafness), motor impairment (e.g., generalized hypotonus, spasticity, or paralysis), or cognitive (e.g., aphasia, apraxia, agnosia) disturbance can conspire to confound accurate diagnostic assessment, constitute a bias to the behavioral assessment, and therefore decrease the probability to observe signs of consciousness. Finally, the environment in which the patient is evaluated may bias assessment findings. Paralytic and sedating medications, restricted range of movement stemming from restraints and immobilization techniques, poor positioning and excessive ambient noise, heat or light can decrease or distort voluntary behavioral responses.

Some sources of error can be avoided, but this is not always possible or within the examiner's control. This is particularly troubling as clinical management, from treatment of pain to end-oflife decision-making, often depends on behavioral observations. To address this problem, neuroimaging procedures have begun to assume an adjunctive role in the diagnostic assessment of patients with DOC.

\section{Functional neuroimaging}

Functional neuroimaging techniques such as positron emission tomography (PET) and 
functional magnetic resonance imaging (fMRI) can provide an objective index of brain activity at rest and during active cognitive processing. Thus, these techniques are well equipped to identify covert cognitive processes in patients who are otherwise incapable of intelligible or sustained behavioral expression, and offer complementary information to bedside examination findings.

In vegetative patients, brain hypometabolism appears similar to individuals in coma, with a $50-60 \%$ decreased global metabolic rate relative to healthy individuals. Hypometabolic activity is further reduced to $60-70 \%$ in patients in "permanent vegetative state" (Tommasino et al., 1995). In VS, the frontoparietal network, including the parietal, mesio-frontal, prefrontal, parieto-temporal, precuneus, and posterior cingular cortex are disproportionately disturbed (Laureys, 2004; Laureys et al., 2004a). In MCS, these regions remain relatively well preserved and their functional connectivity is generally retained (Laureys et al., 2000). Prior investigations have demonstrated that, in VS, auditory or nociceptive stimuli activate primary cortices only, suggesting failure to integrate this information and consequently, absence of conscious perception (Laureys, 2005).

The metabolic pattern differs significantly for patients in MCS. In spite of a global hypometabolism measured at $20-40 \%$ of normal (Schiff et al., 2005), the activity in precuneus and posterior cingular cortex (the most activated regions during wakefulness and the least activated under general anesthesia or during deep slow sleep) was greater as compared to rates noted in patients diagnosed with VS (Laureys et al., 2005b). Similar findings have been noted in functional imaging studies employing auditory or noxious stimulation (Bekinschtein et al., 2004; Boly et al., 2004, 2008; Laureys et al., 2004b; Schiff et al., 2005). Perhaps most importantly, there is evidence that patients in MCS retain higher functional connectivity between the secondary auditory cortex and prefrontotemporal associative cortices (Boly et al., 2005), corroborating the expectation that information processing is more highly integrated in MCS relative to VS.

\section{Case report (AZ)}

A 20-year-old right-handed college student (referred to here as AZ) was admitted for a course of acute neurorehabilitation approximately 3 months after sustaining a severe hypoxicischemic brain injury related to cardio-respiratory arrest caused by a drug overdose. He was found pulseless by at the scene by the emergency medical team and required cardiopulmonary resuscitation for $10 \mathrm{~min}$ before cardioversion was achieved. The initial CT scan was normal but a follow-up scan on day two showed global white matter ischemic changes. The acute medical course was complicated by central fevers and recurrent infections. Medical records from the acute care setting noted that the patient remained unresponsive across the 3-month course, however, family members reported observing episodes of simple command-following and occasional periods of appropriate laughter.

On admission to the rehabilitation unit, flexion contractures were noted in all four extremities (upper greater than lower) and there was no spontaneous purposeful movement. Arousal was well maintained and the auditory startle reflex was intact but there was no evidence of auditory localization. On formal command-following trials using the CRS-R, there was questionable movement of the right toes, however, these responses could not be clearly differentiated from random movement, and there was no other indication of proximal or axial movement to command. To further investigate verbal comprehension in the setting of severe contractures, vocalization commands were administered (i.e., "say ah"). Vocalizations were noted in association with increased oral movement, despite the absence of any spontaneous vocalizations prior to presentation of the commands. No evidence of verbal or gestural communication was observed in response to simple yes/no questions. Assessment of visuoperceptual functions failed to reveal any evidence of object recognition (via eye gaze) and there was a single documented episode of visual pursuit in response to horizontal and vertical movement of a mirror. Noxious stimulation applied to the upper extremities produced facial 
grimace and slight flexion of both lower extremities only.

Over the course of the next 10 months, AZ was evaluated weekly using the JFK CRS-R. Arousal (i.e., eye-opening) was generally well maintained and, despite anecdotal reports of occasional visual fixation, there were no documented episodes of either fixation or pursuit on formal examination. Active and passive range of movement remained severely compromised due to increased tone and spasticity involving all four extremities. The severity of the neuromuscular impairment placed significant constraints on the assessment of command-following. There were, however, infrequent reports of command-following by family members and treating staff, although these behaviors could not be replicated on standardized assessments performed during the 10-month observational period. Incomprehensible vocalizations and crying episodes were frequently noted, but there were no intelligible verbalizations or discernible gestural communication signs at any time. Examination findings were most compatible with VS, although diagnostic certainty was low in view of the occasional manifestation of behaviors associated with conscious awareness. Table 3 shows AZ's CRS-R subscale scores on admission and on follow-up at 3, 6, and 11 months postonset.

In light of the characteristically infrequent, inconsistent, and qualitatively ambiguous signs of consciousness noted in this case, AZ was enrolled in an IRB-approved fMRI study designed to investigate neurophysiologic changes induced by exposure to meaningful sensory stimuli in patients with DOC. Specialized "passive-stimulation" paradigms were administered to monitor changes in cortical networks associated with language and visual processing (Hirsch et al., 2001). In the passive language paradigm, AZ listened to familiar personal stories recounted by a family member (e.g., vacation, wedding). Familiar voices and events were employed to facilitate sustained attention. Thereafter, he was exposed to a second condition in which the narratives were timereversed rendering them unintelligible. Results revealed robust language-specific activation during both the forward and reversed conditions, mirroring previously reported findings in healthy volunteers (Schiff et al., 2005). Extensive clusters of activity were observed extending bilaterally over the transverse temporal gyrus, the middle and superior temporal gyrus, and portions of the precentral and postcentral gyrus bilaterally. More importantly, there were several clusters of activity unique to the forward speech condition observed following subtraction of the reversed from the forward condition. Specific areas of activity tied to high-level language processing included the left superior temporal gyrus (i.e., Wernicke's area), the left supramarginal and superior frontal gyri, and the right medial frontal gyrus. Unexpectedly, both conditions also elicited activity in the occipital cortex (cuneus and lingual gyrus), raising the possibility of language comprehension accompanied by visual imagery (see Fig. 2).

A second "passive viewing" paradigm was presented to engage the visual processing network. A series of back-projected visual images were presented under three conditions. Condition 1 consisted of a combination of familiar (i.e., family members and close friends) and unfamiliar faces, condition 2 was comprised of landscape scenes, and condition 3 utilized flashing checkerboards. AZ was exposed to these three forms of visual stimuli to gauge the selectivity of the activation as well as the degree of preservation of the central nodes comprising this system. Prior studies with healthy volunteers have demonstrated that while each of these stimuli produces visual network activity, faces and landscapes activate mutually exclusive structures (i.e., fusiform face area and parahippocampal place area, respectively) (Epstein et al., 1999; Kanwisher et al., 1997). Findings showed strong activation of the primary visual cortex bilaterally across all three conditions. Of more importance, facial stimuli engaged the fusiform face area of the right inferior temporal gyrus as well as the lingual gyrus bilaterally and right precuneus, consistent with high-level processing of faces. The landscapes elicited activity in the parahippocampal gyrus, lingual gyrus bilaterally, and bilateral precuneus, approximating the response observed in healthy volunteers exposed to landscape scenes (see Fig. 3). 
Table 3. CRS-R response profile on admission to rehabilitation and 3, 6, and 11 months post-injury in patient "AZ," a 20-year-old male with severe anoxic encephalopathy

JFK Coma Recovery Scale - Revised (record form)

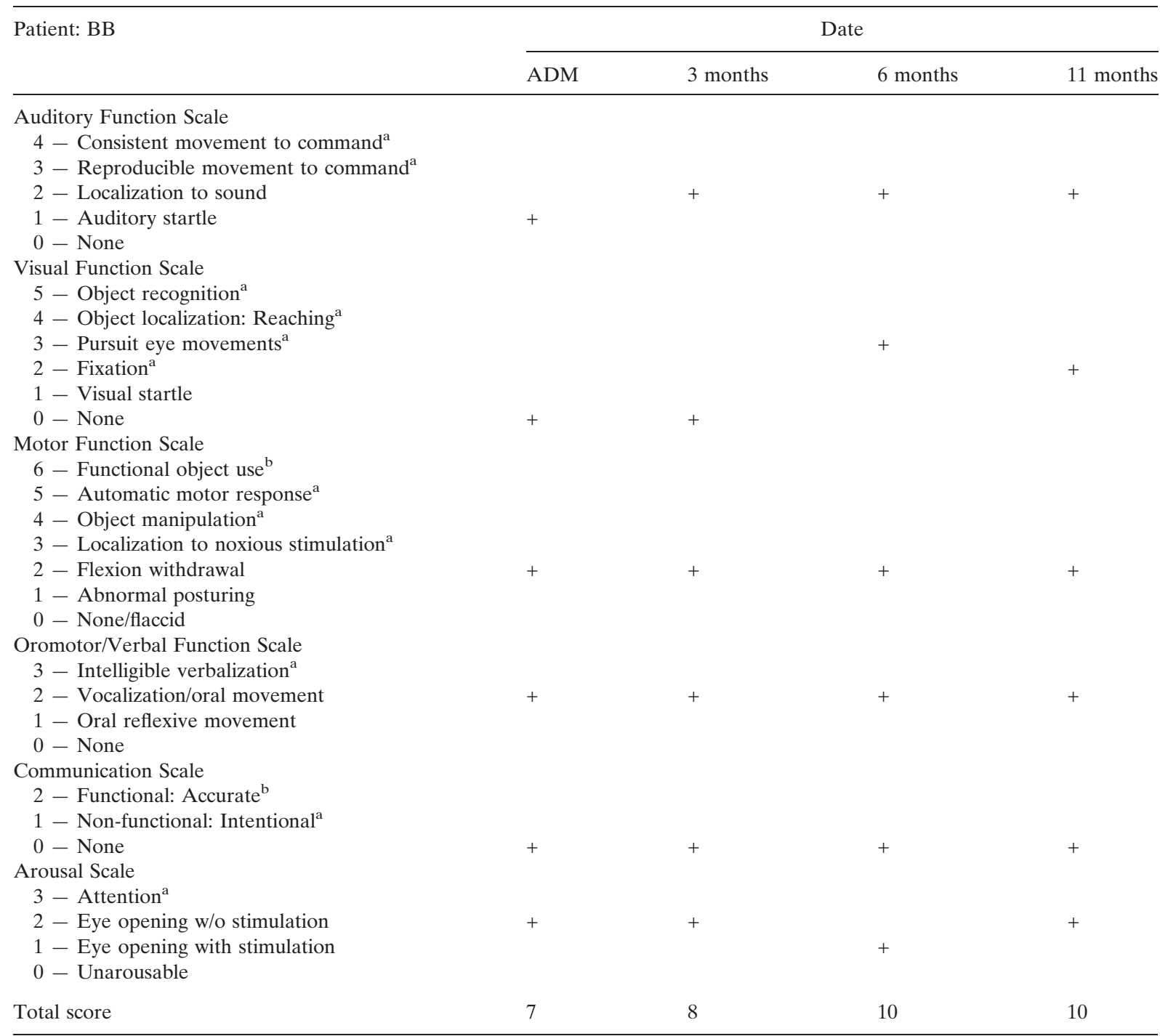

${ }^{\mathrm{a}}$ Denotes MCS.

${ }^{\mathrm{b}}$ Denotes emergence from MCS.

\section{What do these findings mean?}

The results of these studies suggest that some commonly held notions about brain-behavior relationships should be revisited in this patient population. Perhaps most importantly, they clearly illustrate the wide discrepancy that may exist between observable behavior and the underlying neurophysiologic processes believed to support cognitive processing. Such findings also force us to consider the unsettling possibility that cognitive function may be at least partially 

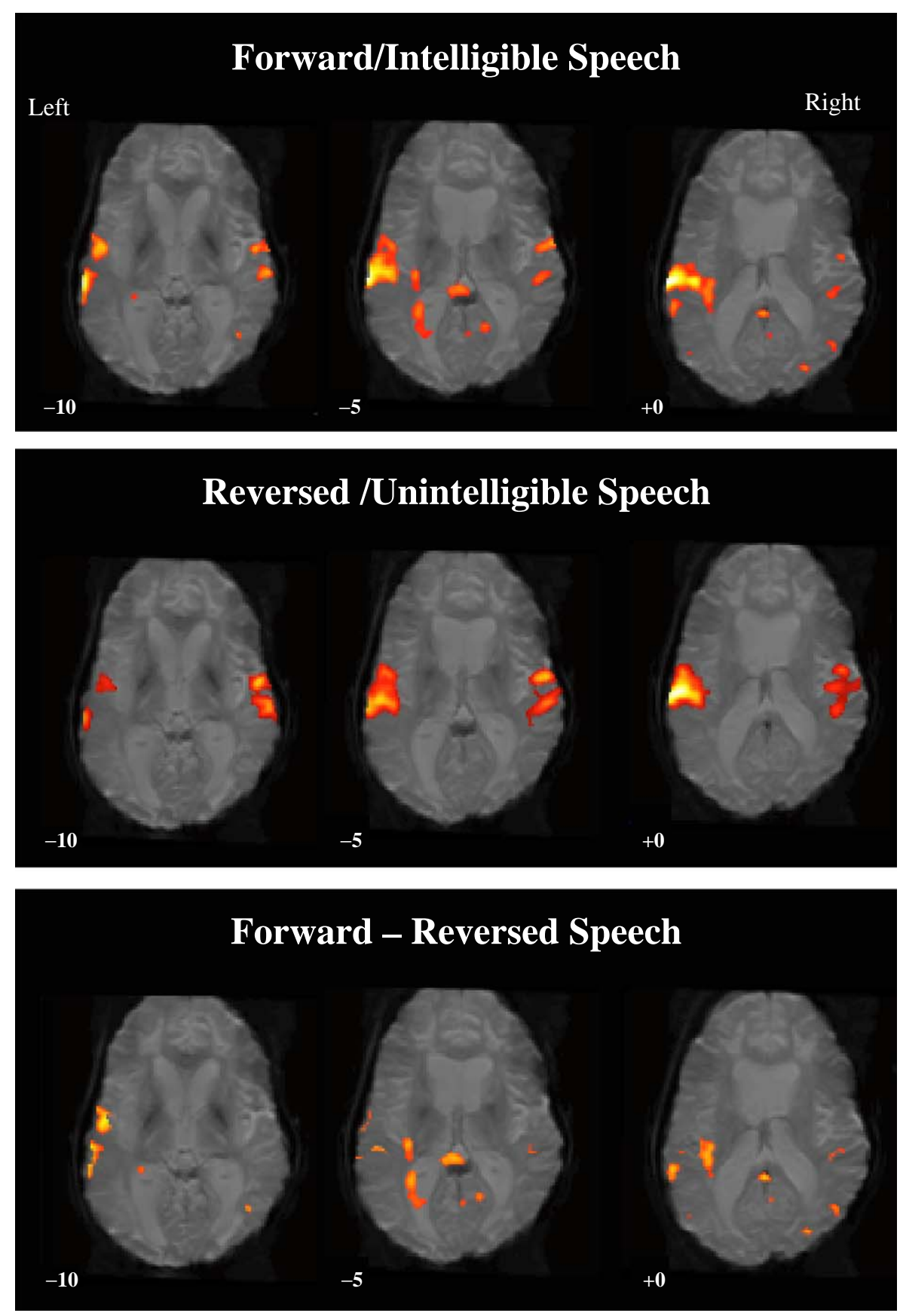

Fig. 2. Regions of activation noted in patient $A Z$ when exposed to spoken narratives. The top panel shows robust activation in left temporal association cortex observed during presentation of comprehensible speech. In the middle panel, regions of activation are well maintained during exposure to unintelligible speech. In the bottom panel, several clusters of activity unique to the forward condition are retained following subtraction of the reversed from forward condition, suggesting preservation of high-level language processing. 

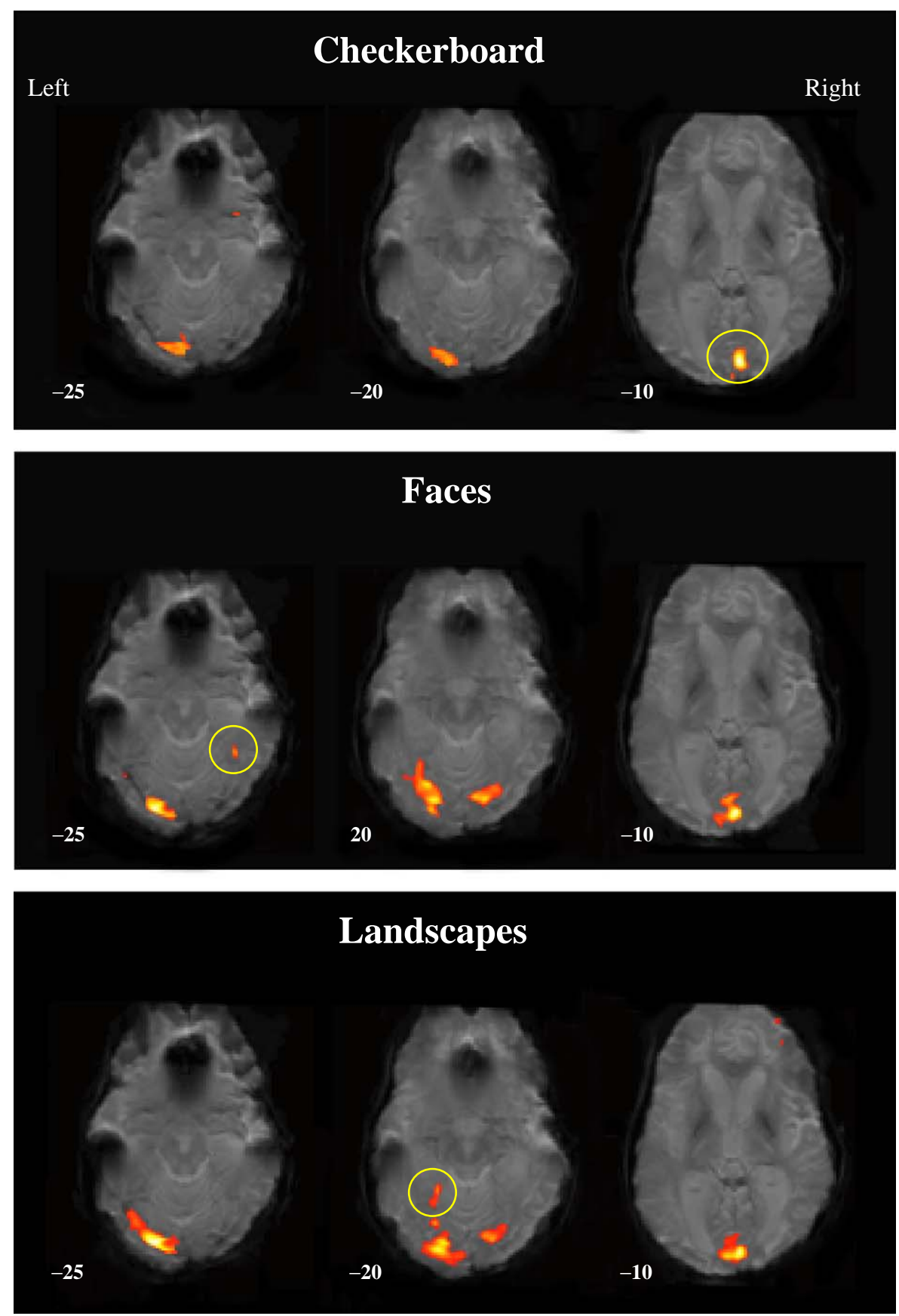

Fig. 3. Regions of activation noted in patient $A Z$ in response to visual stimuli. The top panel shows bilateral activation in the primary cortex observed during exposure to flashing checkerboards. In the middle panel, activation of the fusiform face area is noted during exposure to familiar and unfamiliar faces. The bottom panel shows activation of the parahippocampal place area during presentation of landscape scenes. 
preserved in this case, but lack a mode of expression as the consequence of severely dysfunctional sensory and motor systems. In a sense, these findings may reflect a "functional" LIS. Alternatively, the selective activation of key nodes in the language and visual networks may stem from a more extensively hard-wired neural network than has traditionally been assumed. This premise is supported by recent evidence from electrophysiological and other fMRI activation studies. Employing an event-related cognitive evoked potential paradigm, Perrin et al. (2006) detected a P300 response in three of five patients diagnosed with VS who listened to their own first name versus an unfamiliar name. Similarly, Coleman et al. (2007) found evidence of extensive activation in temporal association cortices in three vegetative patients presented with meaningful speech (i.e., high and low semantically ambiguous sentences) versus unintelligible noise. The contrast between intelligible speech and unintelligible speech sounds provided an opportunity to parse brain regions involved in processing acoustic as well as semantic components of speech from those responsible for processing elementary speech components only. These studies suggest that automatic speech recognition processes mediated by surviving cortical association areas may be preserved in the absence of conscious awareness.

In an effort to circumvent the "automatic versus effortful" processing problem, investigators have relinquished their reliance on passivestimulation paradigms in favor of adopting those that require active processing. Unlike their passive counterparts, active stimulation paradigms direct the subject to perform a cognitive activity on cue. Owen et al. (2006) devised a hierarchical fMRI scanning paradigm in which subjects were directed to imagine either playing tennis or walking around the rooms of their home. Results in normal controls indicated distinct network activation tied to each instructional set. When subjects were instructed to imagine playing tennis, robust activity was observed in the supplementary motor area. In contrast, when subjects were verbally prompted to navigate the rooms of the house, activity shifted to the posterior parietal, parahippocampal gyrus, and lateral premotor regions. Surprisingly, the same findings were observed in a patient whose behavioral profile was reportedly indicative of VS. The authors suggested that, despite the behaviorally unresponsive presentation on bedside examination, the shift in activation patterns coupled to the verbal instructions, constituted evidence that the patient was capable of comprehending language and executing goal-directed behavior. In a similar vein, Schnakers et al. (2008c) recorded eventrelated potentials while subjects either passively listened to their own first name or received instructions to count the number of times they heard their name. The authors reported that healthy controls and patients in MCS, but not those in VS, demonstrated a larger P300 in response to their own name in both the active and passive conditions. The P300 differential was viewed as evidence of intentional compliance with task instructions. As active paradigms such as these continue to be refined, clinicians will be able to convincingly discern whether the capacity for cognitive processing is intact without relying on overt behavior analysis.

Although the standard of the field is now moving toward active paradigms to infer conscious processing, it is unlikely that all patients with DOC will benefit from these paradigms. Those least likely to benefit occupy opposite ends of the severity spectrum. Patients with very little residual brain activity who fail to show activation on passive paradigms (consistent with VS), as well as cases like AZ who demonstrate robust activity (consistent with MCS) but, for unclear reasons are unable to perform effortful tasks, will not be able to engage in active paradigms. Therefore, we believe that both passive and active paradigms will continue to play a role in capturing the full range of cognitive processing capacity characterizing patients with DOC.

\section{Conclusion}

Recovery of consciousness is usually gradual, sometimes marked by emergence of clear behavioral signs, but more often by subtle improvements. Additionally, bedside assessment of 
residual cognitive function is often difficult because of poor arousal, motor impairment, sedating medications, and other confounding factors. Nonetheless, every effort should be made to recognize subtle signs of consciousness as early as possible in the recovery course to avoid misdiagnosis. An accurate diagnosis is crucial not only for daily management (particularly, pain treatment) and end-of-life decisions, but also for prognosis as outcome from MCS is significantly more favorable on average, relative to VS. Knowledge of accepted diagnostic criteria and reliance on validated behavioral assessment scales enhance diagnostic and prognostic accuracy, and facilitate clinical management decisions. Continued development of electrophysiological and functional neuroimaging paradigms designed to detect voluntary brain activity in patients with minimal behavioral output is expected to reduce diagnostic error, increase prognostic specificity, and foster the development of novel interventions to promote recovery.

\section{Acknowledgments}

This study is supported in part by the National Institute on Disability and Rehabilitation Research (Award\# H133A070030), the Belgian National Funds for Scientific Research (FNRS), European Commission, James McDonnell Foundation, Mind Science Foundation, French Speaking Community Concerted Research Action, International Rotary Foundation, Fondation Médicale Reine Elisabeth, and University of Liège.

\section{References}

American Congress of Rehabilitation Medicine. (1995). Recommendations for use of uniform nomenclature pertinent to patients with severe alterations of consciousness. Archives of Physical Medicine and Rehabilitation, 76, 205-209.

Andrews, K., Murphy, L., Munday, R., \& Littlewood, C. (1996). Misdiagnosis of the vegetative state: Retrospective study in a rehabilitation unit. British Medical Journal, 313(7048), 13-16.
Bauer, G., Gerstenbrand, F., \& Rumpl, E. (1979). Varieties of the locked-in syndrome. Journal of Neurology, 221(2), 77-91.

Bekinschtein, T., Niklison, J., Sigman, L., Manes, F., Leiguarda, R., Armony, J., et al. (2004). Emotion processing in the minimally conscious state. Journal of Neurology, Neurosurgery, and Psychiatry, 75(5), 788.

Boly, M., Faymonville, M. E., Peigneux, P., Lambermont, B., Damas, P., Del Fiore, G., et al. (2004). Auditory processing in severely brain injured patients: Differences between the minimally conscious state and the persistent vegetative state. Archives of Neurology, 61(2), 233-238.

Boly, M., Faymonville, M. E., Peigneux, P., Lambermont, B., Damas, F., Luxen, A., et al. (2005). Cerebral processing of auditory and noxious stimuli in severely brain injured patients: Differences between VS and MCS. Neuropsychological Rehabilitation, 15(3-4), 283-289.

Boly, M., Faymonville, M. E., Schnakers, C., Peigneux, P., Lambermont, B., Phillips, C., et al. (2008). Perception of pain in the minimally conscious state with PET activation: An observational study. Lancet Neurology, 7(11), 1013-1020.

Childs, N. L., \& Mercer, W. N. (1996). Late improvement in consciousness after post-traumatic vegetative state. The New England Journal of Medicine, 334(1), 24-25.

Childs, N. L., Mercer, W. N., \& Childs, H. W. (1993). Accuracy of diagnosis of persistent vegetative state. Neurology, 43(8), 1465-1467.

Choi, S. C., Barnes, T. Y., Bullock, R., Germanson, T. A., Marmarou, A., \& Young, H. F. (1994). Temporal profile of outcomes in severe head injury. Journal of Neurosurgery, 81(2), 169-173.

Coleman, M. R., Rodd, J. M., Davis, M. H., Johnsrude, I. S., Menon, D. K., Pickard, J. D., et al. (2007). Do vegetative patients retain aspects of language comprehension? Evidence from fMRI. Brain, 130(Pt. 10), 2494-2507.

Epstein, R., Harris, A., Stanley, D., \& Kanwisher, N. (1999). The parahippocampal place area: Recognition, navigation, or encoding? Neuron, 23(1), 115-125.

Facco, E., Zucchetta, P., Munari, M., Baratto, F., Behr, A. U., Gregianin, M., et al. (1998). 99mTc-HMPAO SPECT in the dia-

gnosis of brain death. Intensive Care Medicine, 24(9), 911-917.

Fins, J. J., Schiff, N. D., \& Foley, K. M. (2007). Late recovery from the minimally conscious state: Ethical and policy implications. Neurology, 68(4), 304-307.

Giacino, J., Ashwal, S., Childs, N., Cranford, R., Jennett, B., Katz, D. I., et al. (2002). The minimally conscious state: Definition and diagnostic criteria. Neurology, 58(3), 349-353.

Giacino, J., \& Kalmar, K. (1997). The vegetative and minimally conscious states: A comparison of clinical features and functional outcome. The Journal of Head Trauma Rehabilitation, 12(4), 36-51.

Giacino, J., Kalmar, K., \& Whyte, J. (2004). The JFK Coma Recovery Scale-Revised: Measurement characteristics and diagnostic utility. Archives of Physical Medicine and Rehabilitation, 85(12), 2020-2029. 
Giacino, J. T., Kezmarsky, M. A., DeLuca, J., \& Cicerone, K. D. (1991). Monitoring rate of recovery to predict outcome in minimally responsive patients. Archives of Physical Medicine and Rehabilitation, 72(11), 897-901.

Giacino, J. T., \& Trott, C. T. (2004). Rehabilitative management of patients with disorders of consciousness: Grand rounds. The Journal of Head Trauma Rehabilitation, 19(3), 254-265.

Giacino, J., \& Whyte, J. (2005). The vegetative and minimally conscious states: Current knowledge and remaining questions. The Journal of Head Trauma Rehabilitation, 20(1), $30-50$.

Gill-Thwaites, H. (1997). The Sensory Modality Assessment Rehabilitation Technique - A tool for assessment and treatment of patients with severe brain injury in a vegetative state. Brain Injury, 11(10), 723-734.

Gill-Thwaites, H., \& Munday, R. (2004). The sensory modality assessment and rehabilitation technique (SMART): A valid and reliable assessment for vegetative state and minimally conscious state patients. Brain Injury, 18(12), 1255-1269.

Hirsch, J., Kamal, A., Moreno, D., Petrovich, N., Giacino, J., Plum, F., et al. (2001). fMRI reveals intact cognitive systems for two minimally conscious patients. Society for Neuroscience, Abstracts, 271, 1397.

Jennett, B. (2005). Thirty years of the vegetative state: Clinical, ethical and legal problems. Progress in Brain Research, 150, 537-543.

Kanwisher, N., McDermott, J., \& Chun, M. M. (1997). The fusiform face area: A module in human extrastriate cortex specialized for face perception. Journal of Neuroscience, 17(11), 4302-4311.

Lammi, M. H., Smith, V. H., Tate, R. L., \& Taylor, C. M. (2005). The minimally conscious state and recovery potential: A follow-up study 2 to 5 years after traumatic brain injury. Archives of Physical Medicine and Rehabilitation, 86(4), 746-754.

Laureys, S. (2004). Functional neuroimaging in the vegetative state. NeuroRehabilitation, 19(4), 335-341.

Laureys, S. (2005). The neural correlate of (un)awareness: Lessons from the vegetative state. Trends in Cognitive Sciences, 9(12), 556-559.

Laureys, S., Faymonville, M. E., Luxen, A., Lamy, M., Franck, G., \& Maquet, P. (2000). Restoration of thalamocortical connectivity after recovery from persistent vegetative state. Lancet, 355(9217), 1790-1791.

Laureys, S., Owen, A. M., \& Schiff, N. D. (2004a). Brain function in coma, vegetative state, and related disorders. Lancet Neurology, 3(9), 537-546.

Laureys, S., Pellas, F., Van Eeckhout, P., Ghorbel, S., Schnakers, C., Perrin, F., et al. (2005a). The locked-in syndrome: What is it like to be conscious but paralyzed and voiceless? Progress in Brain Research, 150, 495-511.

Laureys, S., Perrin, F., Faymonville, M. E., Schnakers, C., Boly, M., Bartsch, V., et al. (2004b). Cerebral processing in the minimally conscious state. Neurology, 63(5), 916-918.

Laureys, S., Perrin, F., Schnakers, C., Boly, M., \& Majerus, S. (2005b). Residual cognitive function in comatose, vegetative and minimally conscious states. Current Opinion in Neurology, 18(6), 726-733.

Majerus, S., Gill-Thwaites, H., Andrews, K., \& Laureys, S. (2005). Behavioral evaluation of consciousness in severe brain damage. In S. Laureys (Ed.), The boundaries of consciousness: Neurobiology and neuropathology (Vol. 150, pp. 397-413). Amsterdam: Elsevier.

Majerus, S., \& Van der Linden, M. (2000). Wessex Head Injury Matrix and Glasgow/Glasgow-Liège Coma Scale: A validation and comparison study. Neuropsychological Rehabilitation, 10(2), 167-184.

McNett, M. (2007). A review of the predictive ability of Glasgow Coma Scale scores in head-injured patients. The Journal of Neuroscience Nursing, 39(2), 68-75.

Medical Consultants on the Diagnosis of Death. (1981). Guidelines for the determination of death. Report of the medical consultants on the diagnosis of death to the President's Commission for the Study of Ethical Problems in Medicine and Biomedical and Behavioral Research. Journal of the American Medical Association, 246(19), 2184-2186.

Owen, A., Coleman, M., Boly, M., Davis, M. H., Laureys, S., \& Pickard, J. (2006). Detecting awareness in the vegetative state. Science, 313(5792), 1402.

Perrin, F., Schnakers, C., Schabus, M., Degueldre, C., Goldman, S., Bredart, S., et al. (2006). Brain response to one's own name in vegetative state, minimally conscious state, and locked-in syndrome. Archives of Neurology, 63(4), 562-569.

Plum, F., \& Posner, J. B. (1966). The diagnosis of stupor and coma (1st ed.). Philadelphia: F.A. Davis.

Plum, F., \& Posner, J. B. (1983). The diagnosis of stupor and coma (3rd ed.). Philadelphia: F.A. Davis.

Schiff, N., Ribary, U., Plum, F., \& Llinás, R. (1999). Words without mind. Journal of Cognitive Neuroscience, 11(6), 650-656.

Schiff, N. D., Rodriguez-Moreno, D., Kamal, A., Kim, K. H., Giacino, J. T., Plum, F., et al. (2005). fMRI reveals largescale network activation in minimally conscious patients. Neuro-

$\log y, 64(3), 514-523$.

Schnakers, C., Giacino, J., Kalmar, K., Piret, S., Lopez, E., Boly, M., et al. (2006). Does the FOUR score correctly diagnose the vegetative and minimally conscious states? Annals of Neurology, 60(6), 744-745. author reply 745.

Schnakers, C., Majerus, S., Giacino, J., Vanhaudenhuyse, A., Bruno, M. A., Boly, M., et al. (2008a). A French validation study of the Coma Recovery Scale-Revised (CRS-R). Brain Injury, 22(10), 786-792.

Schnakers, C., Majerus, S., Goldman, S., Boly, M., Van Eeckhout, P., Gay, S., et al. (2008b). Cognitive function in the locked-in syndrome. Journal of Neurology, 255(3), 323-330.

Schnakers, C., Perrin, F., Schabus, M., Majerus, S., Ledoux, D., Damas, P., et al. (2008c). Voluntary brain processing in disorders of consciousness. Neurology, 71(20), 1614-1620.

Schnakers, C., Vanhaudenhuyse, A., Giacino, J., Ventura, M., Boly, M., Majerus, S., et al. (2009). Diagnostic accuracy of 
the vegetative and minimally conscious state: Clinical consensus versus standardized neurobehavioral assessment. BMC Neurology, 9, 35.

Shiel, A., Horn, S. A., Wilson, B. A., Watson, M. J., Campbell, M. J., \& McLellan, D. L. (2000). The Wessex Head Injury Matrix (WHIM) main scale: A preliminary report on a scale to assess and monitor patient recovery after severe head injury. Clinical Rehabilitation, 14(4), 408-416.

Smart, C. M., Giacino, J. T., Cullen, T., Moreno, D. R., Hirsch, J., Schiff, N. D., et al. (2008). A case of locked-in syndrome complicated by central deafness. Nature Clinical Practice. Neurology, 4(8), 448-453.

Teasdale, G., \& Jennett, B. (1974). Assessment of coma and impaired consciousness. A practical scale. Lancet, 2(7872), 81-84.

The Multi-Society Task Force on PVS. (1994). Medical aspects of the persistent vegetative state (1). The New England Journal of Medicine, 330(21), 1499-1508.

Tommasino, C., Grana, C., Lucignani, G., Torri, G., \& Fazio, F. (1995). Regional cerebral metabolism of glucose in comatose and vegetative state patients. Journal of Neurosurgical Anesthesiology, 7(2), 109-116.

Vanhaudenhuyse, A., Schnakers, C., Bredart, S., \& Laureys, S. (2008). Assessment of visual pursuit in post-comatose states: Use a mirror. Journal of Neurology, Neurosurgery, and Psychiatry, 79(2), 223.

Wijdicks, E. F. (2006). Clinical scales for comatose patients: The Glasgow Coma Scale in historical context and the new FOUR Score. Reviews in Neurological Disease, 3(3), 109-117.

Wijdicks, E. F., Bamlet, W. R., Maramattom, B. V., Manno, E. M., \& McClelland, R. L. (2005). Validation of a new coma scale: The FOUR score. Annals of Neurology, 58(4), 585-593.

Wood, R. L. (1991). Critical analysis of the concept of sensory stimulation for patients in vegetative states. Brain Injury, 5(4), 401-409.

Working Party of the Royal College of Physicians. (2003). The vegetative state: Guidance on diagnosis and management. Clinical Medicine, 3(3), 249-254. 